$17^{\text {th }}$ International Congress of Metrology, 13007 (2015)

DOI: $10.1051 /$ metrology / 201513007

(C) Owned by the authors, published by EDP Sciences, 2015

\title{
Error evaluation in reverse engineering of aspherical lenses
}

\author{
Antonio Piratelli-Filho ${ }^{1}$, Nabil Anwer ${ }^{2}$, Charyar Mehdi Souzani ${ }^{2}$, Goran Devedzic ${ }^{3}$ and Rosenda Valdes Arencibia ${ }^{4}$ \\ ${ }^{1}$ Universidade de Brasília, UnB, Faculdade de Tecnologia, Depto. Engenharia Mecânica, Brasília, DF, Brazil \\ ${ }^{2}$ LURPA, École Normale Superieure - ENS Cachan, 61, av. du Président Wilson, Cachan, France \\ ${ }^{3}$ University of Kragujevac, Faculty of Engineering, Kragujevac, Serbia \\ ${ }^{4}$ Universidade Federal de Uberlândia - UFU, Faculdade de Engenharia Mecânica, Uberlândia, MG, Brazil
}

\begin{abstract}
This work presents a methodology to evaluate the reconstruction of the Computer-Aided Design (CAD) model in reverse engineering of an aspherical lens. A glass made lens is used as reference part for the measurements. The reconstruction of the CAD model is explained and the analysis of the deviations between data points measured on the lens surface and theoretical CAD model is presented. The theoretical model was developed through the measurement of data points on the profile of the part using a Coordinate Measuring Machine (CMM) Cantilever type having a probing uncertainty of $3.2 \mu \mathrm{m}$. A $10^{\text {th }}$ order stepwise polynomial regression model was fitted to the data profile with Matlab software and the parameters $\mathrm{K}$ and $\mathrm{R}$ of the lens were determined by analyzing the aspherical theoretical equations. The uncertainty of these parameters was determined using GUM Supplement 1, Monte Carlo simulation. An additional analysis was carried out to compare the measured points against the $\mathrm{CAD}$ model generated with aspherical profile in Catia software.
\end{abstract}

Key Words: aspherical lens; precision; measurement

\section{Introduction}

Aspherical lenses are important part of optical devices thanks to the curvature that avoids image aberration. It substitutes the spherical lenses group with the advantage of reducing the weight and improving performance of the optical system. Manufacture of aspherical lenses are reported as easy but the metrological issues are considered as complicated, from measurement to fitting the geometry [1].

The aspherical lens profile shows a smooth curvature deviation at the borders in respect to the spherical one. It is considered as a freeform surface with the mathematical equation known from literature. Figure (1) shows the aspherical lens with the parameters vertex radius $(R)$, sag $(z)$ and distance from center $(y)$. Eq. (1) presents the general model to represent the aspherical lens, showing the relation among the variables. The first term represents the conical section of the lens, where the conic constant $K$ characterizes the type of curvature, the parameter $c$ is the reciprocal of the vertex radius $R$ $(c=1 / R)$ and $S$ the radial distance from $\mathrm{Z}$ axis, equals to $S=\sqrt{x^{2}+y^{2}}$ in 3D space ( $S=y$ to lens profile). Other parameters are the polynomial coefficients of the high order aspheric terms, $\mathrm{A}_{4}, \mathrm{~A}_{6}, \mathrm{~A}_{8}$ and $\mathrm{A}_{10}$ [1].

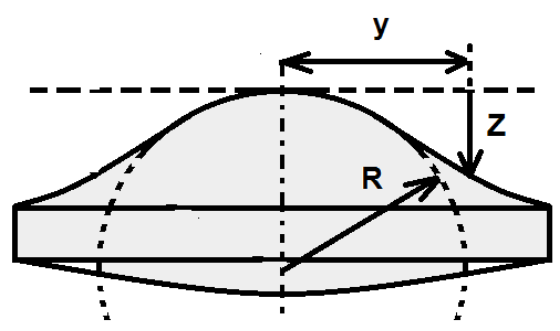

Figure 1. Aspherical lens with design parameters.

$Z=\frac{c S^{2}}{1+\sqrt{1-(K+1) c^{2} S^{2}}}+A_{4} S^{4}+A_{6} S^{6}+A_{8} S^{8}+A_{10} S^{10}$

According to Smith [2], Eq. (1) is flexible and it can represent extreme cases. The author points out that aspheric surfaces have conic sections (paraboloid, ellipsoid, hyperboloid) and can be represented by a power series, as showed by Eq. (2). This equation may be rewritten as a function of $b_{i}$ coefficients and have the formulation according to the Eq. (3).

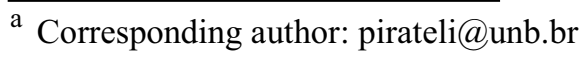




$$
\begin{aligned}
Z=\frac{S^{2}}{2 R} & +\frac{1 \cdot(K+1) \cdot S^{4}}{2^{2} \cdot 2 ! \cdot R^{3}}+\frac{1 \cdot 3 \cdot(K+1)^{2} \cdot S^{6}}{2^{3} \cdot 3 ! \cdot R^{5}}+\frac{1 \cdot 3 \cdot 5 \cdot(K+1)^{3} \cdot S^{8}}{2^{4} \cdot 4 ! \cdot R^{7}}+ \\
& +\frac{1 \cdot 3 \cdot 5 \cdot 7(K+1)^{4} \cdot S^{10}}{2^{5} \cdot 5 ! \cdot R^{9}}+\frac{1 \cdot 3 \cdot 5 \cdot 7 \cdot 9 \cdot(K+1)^{5} \cdot S^{12}}{2^{6} \cdot 6 ! \cdot R^{11}}+\cdots(2)
\end{aligned}
$$

$$
Z=b_{1} S^{2}+b_{2} S^{4}+b_{3} S^{6}+b_{4} S^{8}+b_{5} S^{10}+\cdots
$$

The equation of aspheric profile is used to build the Computer-Aided Design (CAD) model, required to conduct simulation of optical devices using ComputerAided Engineering (CAE) tools. The path followed by light can be simulated and the performance checked. Optical surfaces are demanded in two classes, precision and ophthalmic optics. The difference is associated to the accuracy and tolerances of the lens contour. The tolerance of vertical sag $(z)$ for ophthalmic applications is $\pm 8 \mu \mathrm{m}$, while for surface profile is $\pm 4 \mu \mathrm{m}$, to a lens having $70 \mathrm{~mm}$ in diameter and $60 \mathrm{~mm}$ radius. Other specifications are presented in literature in function of the application (mechanical, electronics or optical) and manufacturing processes [3].

The CAD model is the basis of the Computer-Aided Manufacturing (CAM) processing. The fabrication of aspherical lens can be performed by matrix stamping of polymers using glass molds milled by CAM techniques. The molds are produced by fine grinding in $\mathrm{CNC}$ machines and polishing operation. The aspherical lenses can still be made in glass by $\mathrm{CNC}$ lathe machining with single crystal diamond tools [3]. Brecher et al. (2004) presented a study of aspherical molds machined by using a Non-Uniform Rational B-Splines data interface and concluded that the tool paths were determined with increased flexibility and precision [4].

In search of precision, processing by high temperature compression molding was used to manufacture aspherical micro-lenses in optical glasses [5]. Knowledge of the error sources is a key issue to error compensation and an investigation of the geometric and dynamic machining errors in manufacturing of aspherical surfaces by diamond turning was performed. Error sources as turning parameters (geometry, scale, angular, straightness), machine geometry errors (scale, straightness, angular and squareness) and tool parameters (centering, radius, edge and waviness) were studied [6].

Computational tools are used in the early steps of the optical project to develop the aspherical lens CAD model. Starting from the lens mathematical model, the profile and the surface are designed on computer screen. An alternative for medium and large scale optical components is the acquisition of data from a prototype geometry by measurement, followed by fitting the lens mathematical model to apply reverse engineering techniques. The measurement of aspherical lens has been investigated and a small scale measuring instrument was developed to evaluate micro-aspheric lenses having 1 $\mathrm{mm}$ in diameter with form accuracy smaller than $100 \mathrm{~nm}$ [7].

The research on fitting methods are under way and the literature point directions to be followed. A comparative study was performed with data from a high precision profilometer, comparing fitting algorithms applied to aspherical surface error characterization. These fitting algorithms were the registration and the orthogonal non-linear least squares [8]. A non-linear least squares based algorithm was used to fit aspheric surfaces in 3D space. The authors estimated the parameters of the design equation and discussed the application of the fitting method to measured surfaces [9]. A minimax fitting algorithm was applied to evaluate an ultra-precision aspheric surface and compared with the least squares algorithm [10]. Recently, three fitting algorithms were investigated to evaluate aspherical surfaces form large-volume datasets. The methods of Limited memory-Broyden-Fletcher-Goldfarb-Shanno (L-BFGS), Levenberg-Marquardt (LM) and Iterative Closest Point (ICP) variant were applied and their speed of convergence and robustness was compared [11].

Selection of the measurement instrument and fitting algorithm are determinant of the uncertainty in designed lens. This work presents a methodology to carry out reconstruction of CAD model in reverse engineering of an aspherical lens. A physical lens prototype was used as reference part to take the measurements. The reconstruction of the $\mathrm{CAD}$ model is explained and the analysis of the deviations between lens surface data points and theoretical CAD model is presented. The uncertainty of the aspherical model parameters was determined by Monte Carlo simulation following the GUM Supplement 1 recommendations [12].

\section{Case study}

A reference aspherical lens manufactured in glass and having a diameter $88 \mathrm{~mm}$ at the basis circle was used to carry out the reconstruction of the CAD model. Fig. (2) presents this part, with a dotted line designed over the surface. This line was considered to acquire the profile by measuring data points with a Coordinate Measuring Machine (CMM) Cantilever type, manufactured by Mitutoyo and having a probing error of $3.2 \mu \mathrm{m}$ (uncertainty of point determination), stated by its calibration certificate.

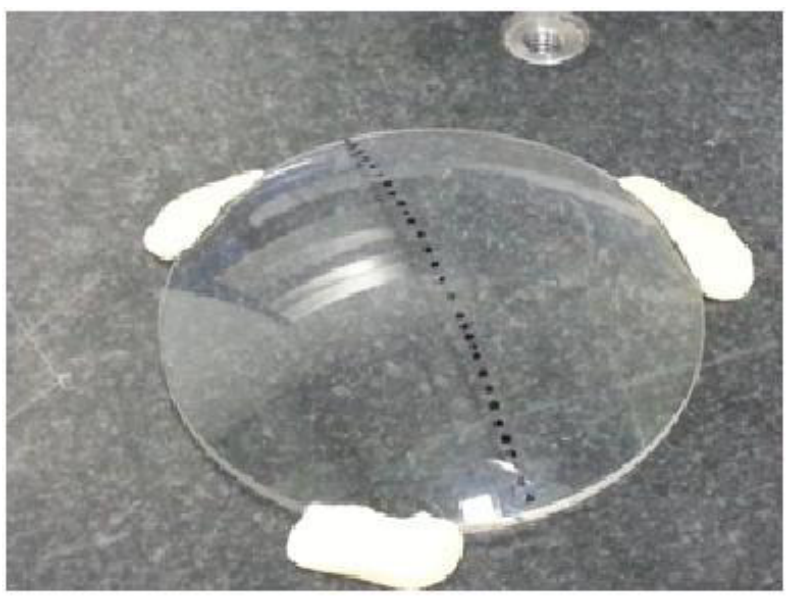

Figure 2. Aspherical lens prototype. 
The measurement strategy is described as follows: i) measurement of the contour circle of the lens taking 30 points after aligning with the machine basis, to determine the circle center; the process was repeated with 5 new measurements of this circle, taking 3 points each time and determining the center to compare with the first measurement. ii) extraction of the orthogonal axis $(\mathrm{Z})$ from the center of the circle, with the CMM software. iii) determination of the top surface point of the lens by moving the CMM probe, locked at the $\mathrm{x}$ and $\mathrm{y}$ axes as determined by the lens circle center. iv) measurement of the lens top surface with the probe to determine the top point, changing the origin of the coordinate system to this point. This strategy is presented in Fig. (3).

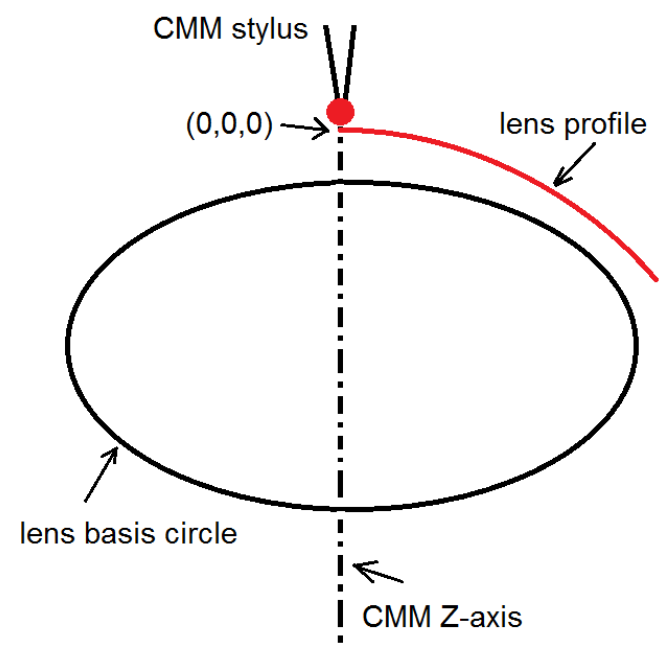

Figure 3. Adopted strategy to determine the center on lens top surface.

The data points were fitted to a curve using an algorithm built in MatLab software. Based on the power series of Eq. (3), a stepwise polynomial equation of $10^{\text {th }}$ order was fitted by least squares algorithm and the coefficients were determined. Hypothesis Student t test was applied to verify which polynomial coefficient was statistically significant and enter in the model at each step. The suitability of the fitted model was verified by applying the $\mathrm{F}$ test over the regression and carrying out a residual analysis, together with the determination parameter $\mathrm{R}^{2}$ [13].

The lens aspherical parameters $K$ and $R$ were determined with the statistically significant coefficients, in association with Eq. (3). The uncertainty of these parameters was simulated by GUM Supplement 1, Monte Carlo simulation, with $\mathrm{M}=10^{5}$ iterations, based on the probing the error of the CMM. The probing error was considered as the limits to simulate variability in $z$ coordinates, whereas the $x$ coordinate was changed in a controlled way and the $y$ coordinate held constant (locked). The variability produced changes and uncertainty in $b_{i}$ coefficients that propagated to the aspherical constants of the lens.

The three-dimensional (3D) CAD model of the surface was developed with Catia software. The model was built by inserting the lens equation with determined parameters $\mathrm{K}$ and $\mathrm{R}$ to initially fit a $2 \mathrm{D}$ line and thus extrude in 3D space to obtain the surface. An analysis of the CAD model was carried out by measuring the lens prototype and taking a cloud of points to compare. It was used the same Cantilever CMM. The cloud of points were compared with the CAD model and the deviations were determined and saved as txt data files. Alignement was accomplished by using the lens external circle of CAD model.

\section{Results and discussion}

A group of 51 points obtained of the aspherical profile was determined with the CMM and the coordinates $\mathrm{x}, \mathrm{y}$ and $z$ were saved in a file txt format. The coordinates of $\mathrm{x}$ and $\mathrm{z}$-axis are used to fit the regression model, since the y coordinates were fixed (locked y-axis). Despite of this, y presented a small variation and was considered to determine the s parameter in the model, as squared root of $x$ squared plus y squared. The results were fitted using the stepwise polynomial regression method with the algorithm developed in MatLab, showed in Table 1. The terms were consecutively introduced in the model, as $\mathrm{x}^{2}$, $\mathrm{x}^{4}, \mathrm{x}^{6}, \mathrm{x}^{8}$ and $\mathrm{x}^{10}$, followed by an evaluation of the regression fitness. This evaluation was done by $t$ tests of the coefficients and by F Snedecor test over regression. Since the term in significant, a new one is introduced and the regression is evaluated again, This process was accomplished until no other term can enter the model.

Table 2 presents the results with the regression coefficients and p-value of t-Student test for the coefficients, after finishing the iteration in stepwise regression. As observed, the coefficients $b_{1}, b_{2}$ and $b_{3}$ were significant at the p-significance level showed, greater than 0.01 or $99 \%$ probability. Thus, the terms of $2^{\text {nd }}, 4^{\text {th }}$ and $6^{\text {th }}$ order in equations (2) and (3) can explain the variability of the data obtained. Besides, the determination coefficient $\mathrm{R}^{2}$ was equals 1 and the ANOVA results presented in Table 3 showed that the regression is significant at $99 \%$ probability $\left(\mathrm{F}_{5,10}(99 \%)=\right.$ 5.64). The residual probability plot is showed in Fig.(4) and the hypothesis of normal distribution of the residuals can not be rejected.

Table 1. Algorithm developed for stepwise polynomial regression fitting and analysis.

\footnotetext{
\% REGRESSION - aspherical profile

clc;clear;close all;

$\%$ Data points measured, from file or type $A=[x$ y z $]$--------.

$\%$ Input data matrix $\mathrm{A}$ with columns $\mathrm{x}, \mathrm{y}$ and $\mathrm{z}$ from points --

$\mathrm{x}=\mathrm{A}(:, 1) ; \mathrm{y}=\mathrm{A}(:, 2) ; \mathrm{z}=\mathrm{A}(:, 3) ; \mathrm{zmed}=\operatorname{mean}(\mathrm{z}) ; \mathrm{n}=\operatorname{size}(\mathrm{z})$;

$\mathrm{s}=\operatorname{sqrt}\left(\mathrm{x} .{ }^{\wedge} 2+\mathrm{y} .^{\wedge} 2\right)$

$\%$ STEPWISE POLYNOMIAL regression, b0 b1 b2 b3 b4 ------

$\%$ Model $z=b 1 . x^{\wedge} 2+b 2 . x^{\wedge} 4+b 3 \cdot x^{\wedge} 6+b 4 . x^{\wedge} 8+b 5 . x^{\wedge} 10$

fprintf('STEPWISE regression, parameters b1 b2 b3 b4 b5');

$\mathrm{xx}=\left[\mathrm{x} .^{\wedge} 2 \mathrm{x} .^{\wedge} 4 \mathrm{x} .^{\wedge} 6 \mathrm{x} .^{\wedge} 8 \mathrm{x} .^{\wedge} 10\right] ;$

[be,SE,pval,inmodel,stats,nextstep] $=$ stepwisefit $(\mathrm{xx}, \mathrm{z})$

$\%$ residual analysis for fitted regression

$\mathrm{m}=$ size(behat);xxhat $=\left[\mathrm{x} .^{\wedge} 2 \mathrm{x} .^{\wedge} 4 \mathrm{x} .^{\wedge} 6\right]$;

behat $=[\mathrm{be}(1)$; be(2); be(3)];zest=xxhat*behat;

$\mathrm{R} 2 \mathrm{mltp}=1-\left(\operatorname{sum}\left(\right.\right.$ resid. $\left.\left.^{\wedge} 2\right)\right) /\left(\operatorname{sum}\left(\mathrm{z}-\operatorname{mean}(\mathrm{z}) .^{\wedge} 2\right)\right)$

$\%$ ANOVA regression $F$ test

glreg $=\mathrm{m}(1)-1$;

SQreg=behat'*xxhat'*z-n(1)*zmed ${ }^{\wedge} 2$;
} 
QMreg=SQreg/glreg;

glt=n(1)-1;SQT=z'*z-n(1)*zmed ${ }^{\wedge} 2 ;$ glres=glt-glreg;

SQres=SQT-SQreg;QMres=SQres/glres;

$\mathrm{Fc}=\mathrm{QMreg} / \mathrm{QMres}$

$\%$ RESIDUAL analysis

normplot(resid)

$\%$ END

Table 2. Stepwise polynomial regression results, with parameters and respective p-values. (*significant)

\begin{tabular}{|c|c|c|c|c|c|}
\hline & $\mathrm{b}_{1}(*)$ & $\mathrm{b}_{2}\left(^{*}\right)$ & $\mathrm{b}_{3}(*)$ & $\mathrm{b}_{4}$ & $\mathrm{~b}_{5}$ \\
\hline values & 0.0048138 & $1 \times 10^{-7}$ & $8 \times 10^{-12}$ & $\begin{array}{c}-9 \times 10^{-} \\
16\end{array}$ & $-2 \times 10^{-19}$ \\
\hline p-value & $5 \times 10^{-125}$ & $4 \times 10^{-41}$ & $3 \times 10^{-13}$ & 0.58 & 0.62 \\
\hline
\end{tabular}

Table 3. ANOVA results of the regression fitting.

\begin{tabular}{|c|c|c|c|c|}
\hline SV & SS & DF & MS & $F_{c}$ \\
\hline Regression & 434.2284 & 2 & 217.1142 & $3.4 \times 10^{4}$ \\
\hline Residual & 0.3006 & 47 & 0.0064 & \\
\hline Total & 434.5289 & 49 & & \\
\hline
\end{tabular}

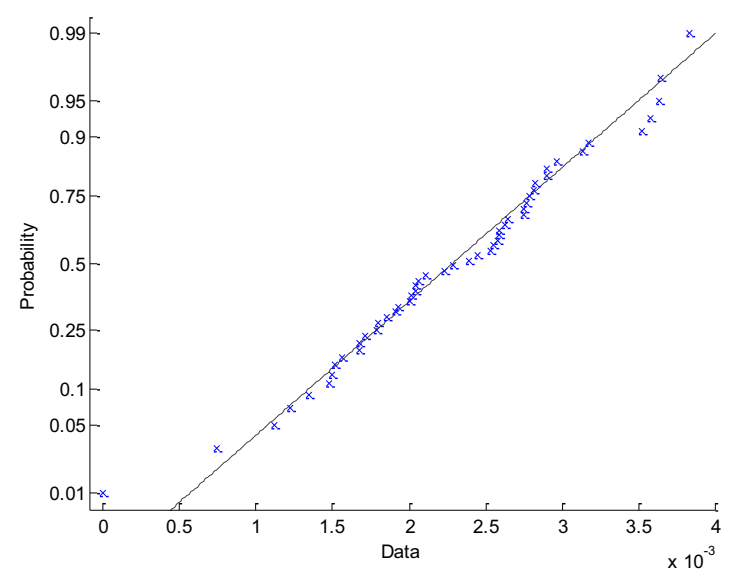

Figure 4. Residual analysis of polynomial regression.

The lens parameters $\mathrm{R}$ and $\mathrm{K}$ were determined by using the equations (2) and (3), using the stepwise polynomial regression coefficients $b_{1}$ and $b_{2}$. The results are showed by equations (4) and (5). These lens parameters were used to generate the aspherical profile as showed in Fig. (5).

$$
\begin{aligned}
& R=0.5 / b_{1}=103.868 \mathrm{~mm} \\
& K=8 . b_{2} \cdot R^{3}-1=-0.081
\end{aligned}
$$

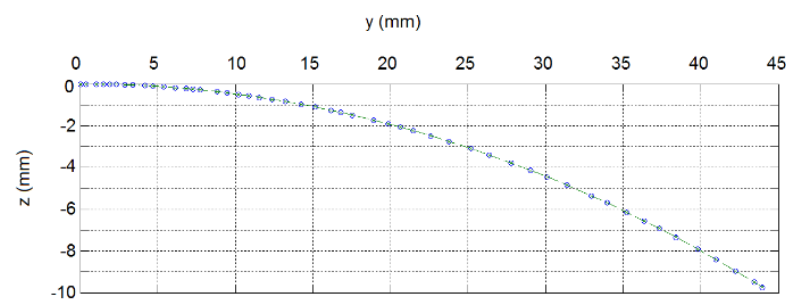

Figure 5. Aspherical profile generated by stepwise polynomial regression with data points.
The uncertainty in parameters $\mathrm{R}$ and $\mathrm{K}$ determined by polynomial regression was carried out by Monte Carlo (MC) simulation with the algorithm developed and showed in Table 4 . The number of iterations was $10^{5}$ and the values of standard and expanded (95\%) uncertainties were determined and presented in Table 5 . Repetition of the simulation procedure showed variability only in the third decimal digit of $\mathrm{R}$ and $\mathrm{K}$ means and the uncertainty at the first decimal digit.

Table 4. Algorithm to determine the uncertainty by Monte Carlo simulation.

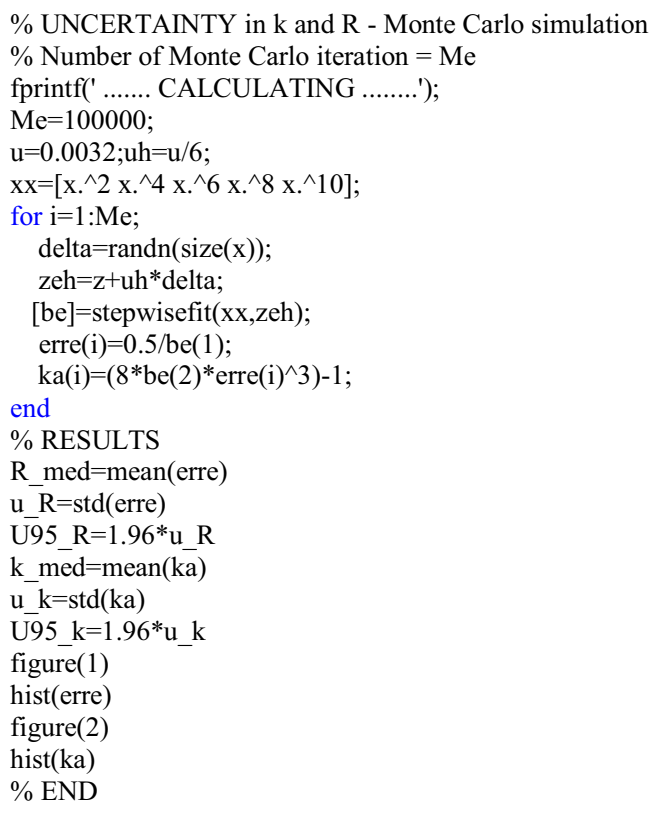

Table 5. Results of MC simulation

\begin{tabular}{|c|c|c|c|c|}
\hline Parameter & $\begin{array}{c}\text { Estimated } \\
\text { (Mean) }\end{array}$ & $\begin{array}{c}\text { Stand. } \\
\text { uncertainty }\end{array}$ & $\begin{array}{c}\text { Expanded } \\
\text { uncertainty }\end{array}$ & $\begin{array}{c}\text { MC } \\
\text { Trials }\end{array}$ \\
\hline $\mathrm{R}$ & 103.869 & 0.025 & 0.049 & $10^{5}$ \\
\hline $\mathrm{K}$ & -0.078 & 0.021 & 0.042 & $10^{5}$ \\
\hline
\end{tabular}

The graphics of Fig. (6) and (7) presents the distribution of the simulated results of $\mathrm{R}$ and $\mathrm{K}$, respectively. It is shown that the distribution of the values is close to the Normal probability distribution. 


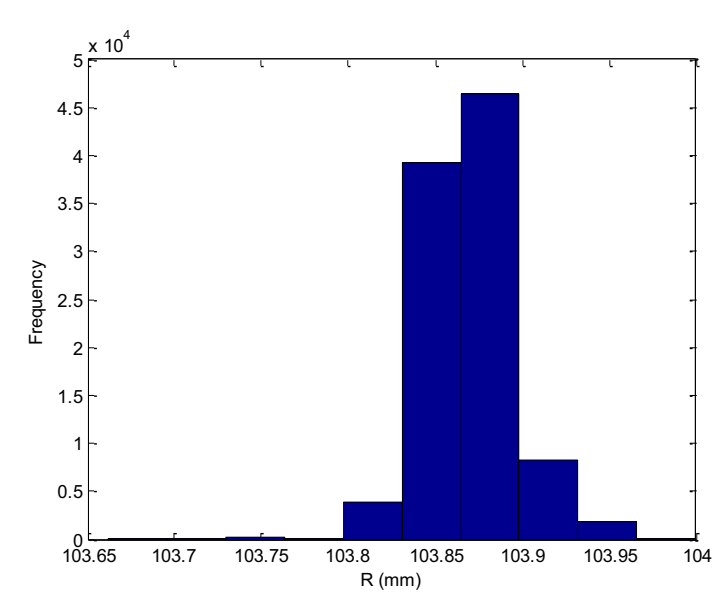

Figure 6. Results of MC simulation for R parameter.

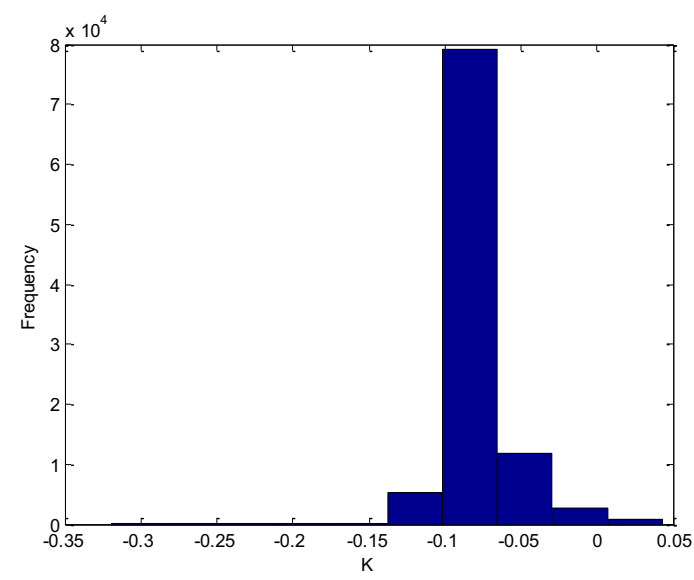

Figure 7. Results of MC simulation for $\mathrm{k}$ parameter.

The profile fitted by regression was used to build the 3D CAD model in Catia Software. A cloud of points of the same aspherical lens prototype was measured with the CMM and the comparison with the CAD model is presented in Fig. (8). The deviations between the points and the CAD model are presented by the hairs (line segments) and are distributed along the entire aspherical surface.

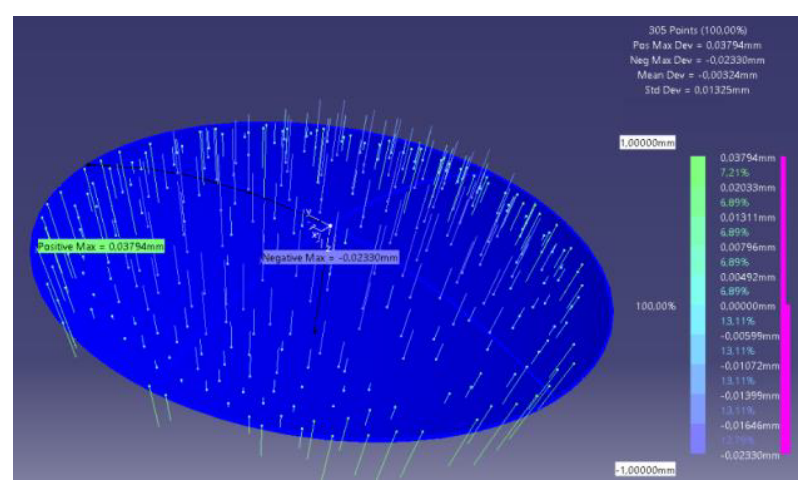

Figure 8. Analysis of the CAD model deviations.

The analysis showed a mean deviation of $-0.003 \mathrm{~mm}$, a standard deviation of $0.013 \mathrm{~mm}$ and a range of 0.061 $\mathrm{mm}(0.038$ to $-0.023 \mathrm{~mm})$. The variability was greater than the CMM uncertainty $(0.0032 \mathrm{~mm})$ but these values have the contribution of other sources as algorithm fitting, measurement errors, surface form deviation, alignment and others.

\section{Conclusions}

The reconstruction methodology applied to aspherical lens was presented and was based on measurement of a lens prototype profile. A detailed statistics analysis was carried out and the parameters $\mathrm{R}$ and $\mathrm{K}$ of the lens were determined by polynomial regression. An uncertainty analysis was performed by Monte Carlo simulation and the standard and expanded (95\%) uncertainties were determined with precision of two decimal digits. CAD model analysis showed a uniform surface with deviations distributed without any bias.

The considerations presented to large aspherical lenses can be extended to small lenses since the replacement of the measuring instruments by others having greater accuracy and reduced uncertainty is accomplished.

\section{References}

[1] D. Malacara, Z. Malacara. Handbook of optical design. Marcel Dekker, $2^{\text {nd }} E d .500$ p (2004)

[2] Smith, W.J. Modern Optical Engineering. McGrawHill, $3^{\text {rd }}$ Ed. 617 p (2000)

[3] V.C. Venkatesh, 2003. Current Science, 84, n.o 9, 1211-1219 (2003)

[4] C. Brecher, M. Weck, M. Winterschladen, S. Lange, O. Wetter, T. Pfeifer, D. Döner, E. Brinksmeier, L. Autschbach. ASPE Winter Meeting (2004)

[5] G.C. Firestone, A.Y. Yi. Applied Optics, 44, n.o 29, 6115-6122 (2005)

[6] A. Sohn, K.P. Garrard, T.A. Dow. ASPE Annual Meeting (2005)

[7] A. Shibuya, Y. Arai, Y. Yoshikawa, W. Gao. ASPE Annual Meeting (2008)

[8] N. El-Hayek, N. Anwer, H. Nouira, O. Gibaru, M. Damak, P. Bourdet. Procedia CIRP, 27, 41-46 (2015)

[9] W. Sun, J.W. McBride, M. Hill. Precision Engineering, 34, 171-179 (2010)

[10] X. Zhang, X. Jiang, P.J. Scott. Journal of Physics: Conference Series, 311 (2011)

[11] N. El-Hayek, H. Nouira, N. Anwer, O. Gibaru, M. Damak. Precision Engineering, 38, 935-947 (2014)

[12] JCGM 101. Evaluation of measurement data Supplement 1 to the GUM (2008)

[13] Y.Dodge and V. Rousson. Analyse de regression appliquée. Dunod, $2^{\text {nd }}$ ed. 279 p. (2004)

\section{Acknowledgements}

The authors would like to acknowledge the Conselho Nacional de Desenvolvimento Científico e Tecnológico $(\mathrm{CNPq})$, Brazil, and the Fundação de Apoio à Pesquisa do Distrito Federal (FAPDF), Brazil, for financing this work. 